\title{
Therapeutic Effects of Curcuma longa against Rotenone-Induced Gross Motor Skill Deficits in Rats
}

\author{
Syeda Madiha, Zehra Batool, Saiqa Tabassum, Laraib Liaquat, Sadia Sadir, \\ Tahira Perveen and Saida Haider*
}

Neurochemistry and Biochemical Neuropharmacology Research Unit, Department of Biochemistry, University of Karachi, Karachi 75270, Pakistan

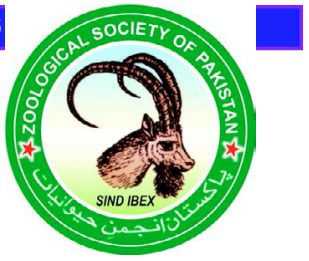

CrossMark

\begin{abstract}
A B S T R A C T
Rotenone (pesticide and mitochondrial complex I inhibitor) has been associated to induce toxicity in humans and as well as in animals. The present study evaluated the protective role of curcumin against rotenone-induced motor deficits, biochemical and neurochemical alterations. Rotenone was injected subcutaneously at the dose of $1.5 \mathrm{mg} / \mathrm{kg}$ for 8 days. Supplementation of curcumin $(100 \mathrm{mg} / \mathrm{kg} / \mathrm{day}$, p.o.) was started before 15 days of rotenone injection. The effects of curcumin pre-treatment were evaluated by different motor behavioral parameters (pole test, Kondziela's inverted screen test, inclined plane test, open field test, beam walking test and footprint test). Biochemical and neurochemical alterations were also monitored. Pre-treatment with curcumin reversed the motor impairments, biochemical and neurochemical alterations which were produced by rotenone. Furthermore, curcumin treatment restored the dopaminergic and cholinergic functions. We conclude that curcumin prevent against toxic effect of rotenone and can be beneficial against different neurodegenerative diseases.
\end{abstract}

Article Information
Received 25 June 2017
Revised 30 August 2017
Accepted 02 October 2017
Available online 11 May 2018
Authors' Contribution
SM and SH designed the research.
LL, SS and SM conducted aboratory
work. ZB and ST performed statistical
analyses. LL, SS, SM and TP wrote the
article. SH supervised the research.
Key words
Curcuma longa, Rotenone, Dopamine,
Acetylcholine, Kondziela's inverted
screen test, Footprint test.

\section{INTRODUCTION}

$\mathrm{E}$ nvironmental factors are the main contributor to the development of neurodegenerative diseases in humans (Freire and Koifman, 2012; Gatto et al., 2009). There are several environmental factors among them the exposure to agricultural chemicals such as pesticide (Zeb et al., 2016) gaining more focus in recent years for the development of human diseases. Rotenone is one of the toxins which extensively used as pesticide; primarily it was used as a fish poison. Epidemiological studies suggested that exposure to environmental toxin such as rotenone has been associated to increase Parkinson's disease (Greenamyre et al., 2011; Freire and Koifman, 2012; Gatto et al., 2009). Rotenone is lipophilic in nature which easily crosses the blood brain barrier and gathers in mitochondria thus, inhibits the mitochondrial complex I (NADH-ubiquinone oxidoreductase) (Betarbet et al., 2000). Complex I function is to translocate proton which is used for maintenance of energy (Hatefi, 1985). Rotenone toxicity causes complex I inhibition which results in the energy crisis in cells and provide important impending into the mechanism of mitochondrial related human diseases.

\footnotetext{
* Corresponding author: saida-h1@hotmail.com 0030-9923/2018/0004-1245 \$ 9.00/0

Copyright 2018 Zoological Society of Pakistan
}

Rotenone causes systemic inhibition of mitochondrial complex I, which results in the formation of reactive oxygen species (ROS) that block the electron transport chain and cause neurodegeneration (Moon et al., 2005). Rotenone can enters into dopaminergic neurons because it does not depend on dopamine (DA) transporters, resulting in complex I inhibition (Betarbet et al., 2000). Although, rotenone is widely distributed in the brain, it can cause selective neurodegeneration in specific regions (Greenamyre et al., 2011), and has been directly associated with the motor impairments in rats. It is reported that rotenone administration in rats caused the death of dopaminergic neurons (Betarbet et al., 2000). The bulk of the data are suggesting that rotenone exposure contributes to toxic effects in both human and animals and that can be used to test potential compounds for possible therapeutics.

Curcumin is an active polyphenolic compound of turmeric (Curcuma longa) which is commonly used as food additives in Asian cuisine. Curcumin is lipophilic in nature and has multiple characteristics such as anti-inflammatory, antioxidant, anticarcinogenic, and anti-protein aggregate activities (Fu and Kurzrock, 2010; Hatcher et al., 2008). Curcumin provides protection against different toxins. Various studies reported that pre and post-treatment with curcumin cause a significant attenuation of toxin induced depletion in striatal dopamine level and neuronal cell loss (Rajeswari, 2006; Vajragupta et al., 2003; Zbarsky et al., 
2005). A number of pharmacological effects of curcumin have been studied, however effects of curcumin on rotenone-induced toxicity is still not clear. Therefore the main goal of the study was to investigate the protective effect of curcumin in ameliorating the rotenone-induced motor deficits by using different behavioral parameters (pole test, kondziela inverted screen test, inclined plane test, open field test, beam walking and footprint test) and biochemical alterations in rats. Furthermore, dopaminergic neurotransmission was also analyzed. The emphasis in our study was to find out the cholinergic and dopaminergic activity following rotenone administration and further to determine the protective role of curcumin in restoring the cholinergic and dopaminergic functions in rotenone intoxicated rat.

\section{MATERIALS AND METHODS}

\section{Animals}

Twenty four (24) Albino-Wistar rats (150-200g) were used in the study. Rats were confined separately with access to rodent diet and water (Bocarsly et al., 2012). Animals were allowed to acclimatize for 3 days in order to cancel out the environmental psychological suffering.

\section{Reagents and chemicals}

Rotenone, curcumin and rest of the chemicals were obtained from Sigma Chemical Co. (St. Louis, USA).

\section{Experimental protocol}

After three days of acclimation period, rats $(n=24)$ were divided into four groups and named as control (sunflower oil), curcumin (Cur), rotenone (Rot) and Curcumin+Rotenone (Cur+Rot) each group containing six rats $(n=6)$. Treatment was started with the administration of curcumin dissolved in oil at the dose of $100 \mathrm{mg} / \mathrm{kg}$ to Cur and Cur+Rot groups. Sunflower oil was given to control group. Remaining groups were given tap water orally with the same procedure. After 15 days of curcumin administration, rotenone was injected subcutaneously (s.c) to Rot and Cur+Rot groups at the dose of $1.5 \mathrm{mg} /$ $\mathrm{kg}$ dissolved in sunflower oil. Curcumin was given for 15 days prior to the administration of rotenone and along with rotenone for eight days (Madiha et al., 2017). Body weight and food intake was measured throughout the pre-treatment of curcumin and rotenone administration. Rotenone injection was continued for eight days during which curcumin treatment was continued. After the rotenone injection, rats were subjected to open field test, beam walking test, and pole test to observe the motor coordination and balance. Kondziela's inverted screen test and inclined plane test were also carried out to determine the muscular strength and cataleptic effects respectively following rotenone administration. Footprint test was used to attain the foot step pattern of rats (Madiha et al., 2017). After behavioral procedures rats were decapitated in order to collect their brains. Whole brain was taken out from the skull within $30 \mathrm{~s}$ after decapitation. The dissection procedure for dissecting brain regions was carried out as described by Haleem (1990) and Haleem and Perveen (1994). For neurochemical assay all brain samples were kept at $-70^{\circ} \mathrm{C}$ (Fig. 1). Dopamine (DA), dihydroxyphenylacetic acid (DOPAC), ACh (acetylcholine) and AChE (acetylcholinesterase) were estimated in striatum. Reduced glutathione (GSH), malondialdehyde (MDA) levels and enzyme activities were also monitored including superoxide dismutase (SOD), catalase (CAT) and glutathione peroxidase (GPx) in rat brain.

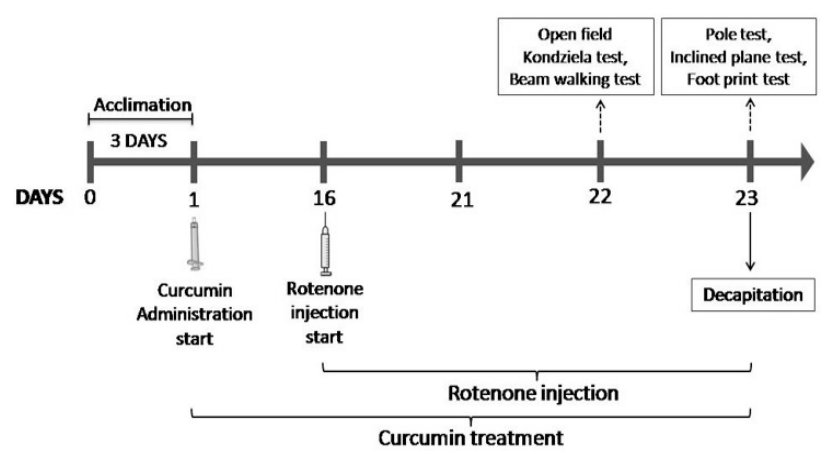

Fig. 1. Schematic representation of experimental protocol.

\section{Behavioral analysis \\ Pole test}

The pole test used to assess basal ganglia-related movement disorders in rodents. In brief, rats were placed head-up on top of a vertical wooden pole $(2.5 \mathrm{~cm}$ in diameter and $100 \mathrm{~cm}$ in height) and base of the pole was positioned in the home cage. The time to come down from pole to the floor was monitored in rats (Yanpallewar et al., 2004).

\section{Kondziela's inverted screen test}

Kondziela the inverted screen test has been used previously for measure of muscular strength of animals using all four limbs (Kondziela, 1964). The test was done by placing the rat in the center of screen which was inverted over 120 seconds with the rats head declining first. The time when the rat fell off from the screen was noted.

\section{Inclined plane test}

The cataleptic effect was investigated according 
to previously described method by Costall and Naylor (1974). The test was done by gently placing the animal on an inclined surface for three minutes and the duration of time the animal remained in one position in seconds was recorded. Cataleptic score was calculated (Batool et al., 2010).

\section{Open field test}

The open field test is used to monitor motor activity. The apparatus consists of a square $(76 \times 76 \mathrm{~cm})$ with walls $42 \mathrm{~cm}$ high. In the central square of the open field animals were placed individually. Latency to move and numbers of square crossed were monitored for five minutes (Haleem et al., 2007).

\section{Beam walking test}

Beam walking is a test of motor coordination. The rats have to cross a beam which is suspended between a start platform and their home cage at a height of $50 \mathrm{~cm}$ and is supported by two pillars. A cushion was placed under the beam in order to protect the animals from the bang into the floor. The difficulty of this task can be assorted by using beams with different shapes and widths (Jover et al., 2006). Motor activity was assessed by the ability of a rat to crossways a different size of beams. Three circular beams of different diameters were used in this study such as $3 \mathrm{~cm}$, $2 \mathrm{~cm}, 1 \mathrm{~cm}$ and length of $100 \mathrm{~cm}$. In the training phase, animals were trained to traverse the three beams (from widest to narrowest). This helps to make certain that the behavior during testing is more stable and more precisely reflects motor coordination as opposed to the rodent's natural aversion to crossing over unprotected spaces. After training session testing phase was done and the time taken to cross the beam was recorded.

\section{Footprint test}

To take the footprints, the rat hindlimb and forelimb were colored with green and red paints. Animals walk on a runway having following dimensions (100 cm length, 10 $\mathrm{cm}$ wide). A white paper was positioned on the floor of the runaway for each rat run. The footprints were evaluated for three step parameters: stride length, base width and overlap between forelimb and hindlimb in centimeters. Four successive steps were chosen for evaluation (NascimentoFerreira et al., 2013).

\section{Neurochemical analysis \\ Determination of monoamines}

Concentrations of DA and its metabolite DOPAC in striatum was assessed by means of high performance liquid chromatography with electrochemical detector (HPLC-EC) as described by Haider et al. (2014).
Determination of striatal acetylcholine (ACh) content and acetylcholinesterase (AChE)

The method as explained by Batool et al. (2016) was used to estimate acetylcholine (ACh) content and the method as illustrated by Haider et al. (2015) was used to determine acetylcholinesterase (AChE) activity in striatum.

\section{Biochemical analysis}

Activities of superoxide dismutase (SOD), catalase (CAT), glutathione peroxidase (GPx) and lipid peroxidation (LPO were estimated in the same way as explained by Haider et al. (2015) whereas reduced glutathione (GSH) was determined as explained by Haider et al. (2016).

\section{Statistical analysis}

Data are presented as mean \pm SD. Mean differences were evaluated by one-way ANOVA followed by Tukey's post-hoc test using SPSS version 20.

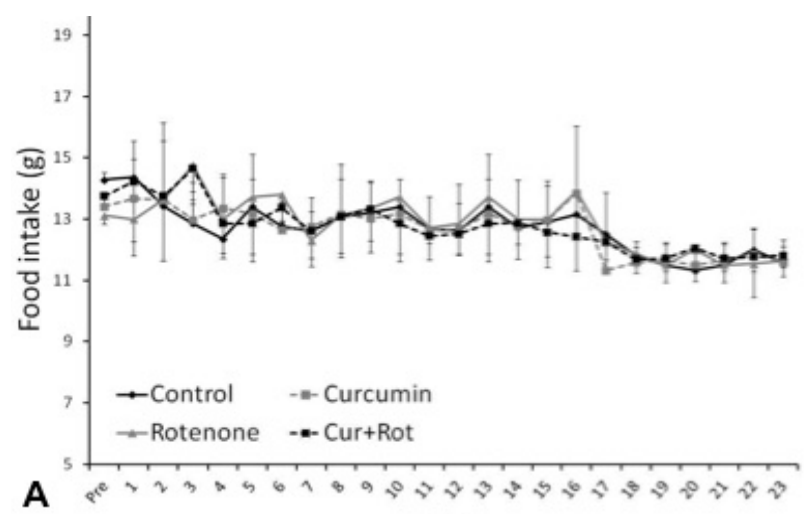

A

Days

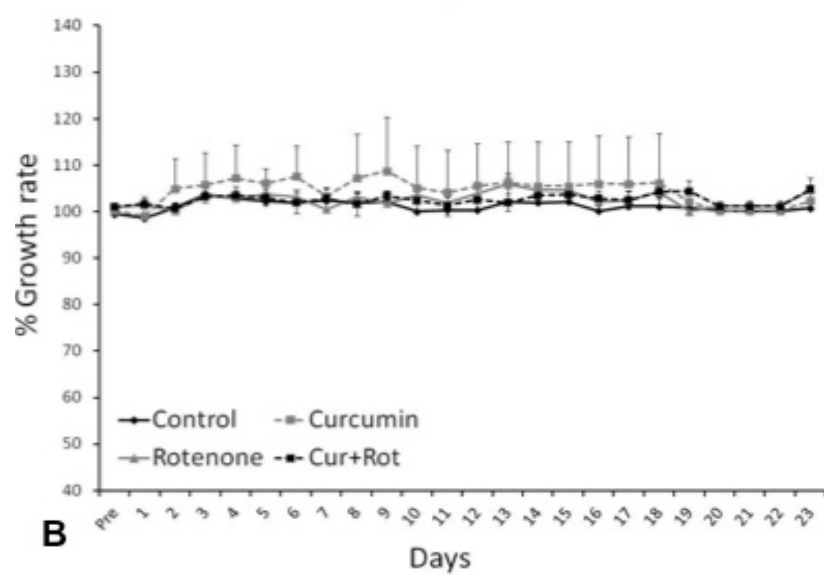

Fig. 2. Effects of rotenone and pre-administration of curcumin on food intake (A) and growth rate (B). Values are mean $\_$SD $(n=6)$. Difference was non-significant obtained by one-way ANOVA following Tukey's test. Growth rate in \% was calculated by Present weight/Initial weight $\mathrm{x}$ 100). 


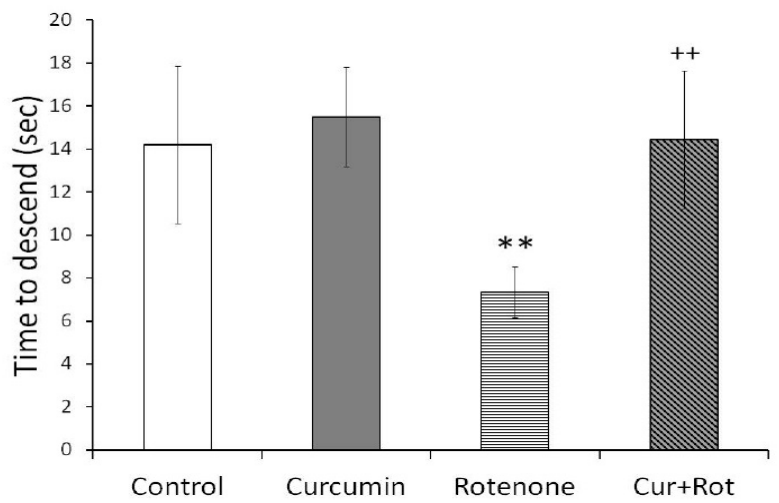

Fig. 3. Rotenone significantly impaired the motor skills in pole test as observed by decreased time to descend indicating reduced muscular strength. This effect was significantly attenuated by pre-administration of curcumin in Cur+Rot group when comparing with rotenone administered rats. Values are mean $\pm \mathrm{SD}(\mathrm{n}=6)$. Significant difference was obtained by one-way ANOVA following Tukey's test. ${ }^{* *} p<0.01$ versus controls and $++p<0.01$ versus rotenone injected group $(n=6)$.

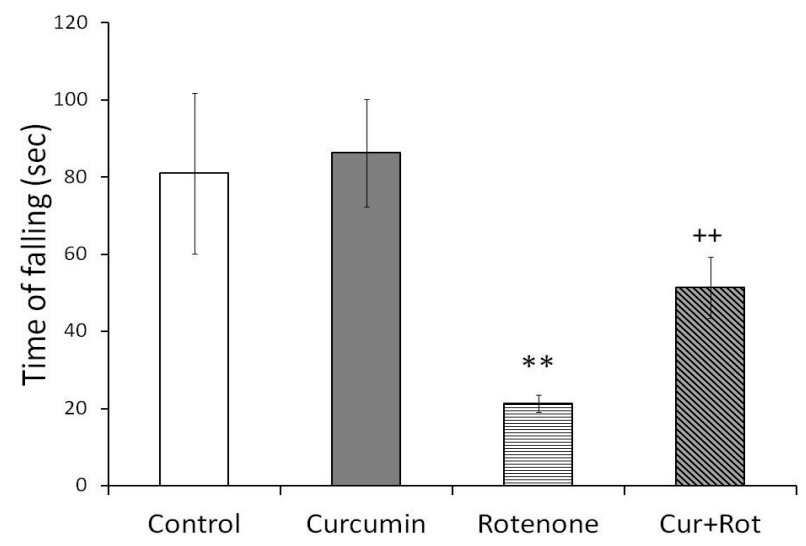

Fig. 4. Effect of curcumin on muscular strength for all four paws was assessed by Kondziela test. Rotenone impaired the muscular strength of rats as observed by significantly decreased time to hold the inverted screen as compared to controls $(* * p<0.01) .15$ days pre-treatment with curcumin attenuated the effects of rotenone on motor coordination as evident by significantly increased inverted screen score in Cur+Rot group as compared to rotenone $(n=6)$ injected rats $(++p<0.01)$. Significant differences were obtained by one-way ANOVA followed by Tukey's test. Values are mean $\pm \mathrm{SD}(\mathrm{n}=6)$.

\section{RESULTS}

\section{Food intake and growth rate}

Food intake and growth rate (Fig. 2A, B) were monitored daily to assess the general health condition of rats after rotenone administration. Following rotenone administration general health was not changed.

\section{Muscular strength}

Pole test (Fig. 3) and Kondziela's inverted screen (Fig. 4) were used to analyze motor and muscular strength following the administration of rotenone and curcumin. One-way ANOVA revealed considerable effects of treatment in pole test $(F=10.91, p<0.01)$ and inverted screen test $(F=30.67, p<0.01)$. Post-hoc analysis by Tukey's test showed a significantly decrease in time to descend the pole in rats injected with rotenone as compared to controls $(p<0.01)$. However, Time to descend was significantly increased in Cur+Rot group $(p<0.01)$ when it compared to rotenone injected group. Kondziela's inverted screen test also showed impaired muscular strength by rotenone administration as evident by significantly reduced time of falling $(p<0.01)$ as compared to that of controls. Whereas, prior administration of curcumin drastically increased the time of falling $(p<0.01)$ from inverted screen in Cur+Rot group when compared to rotenone administered group.

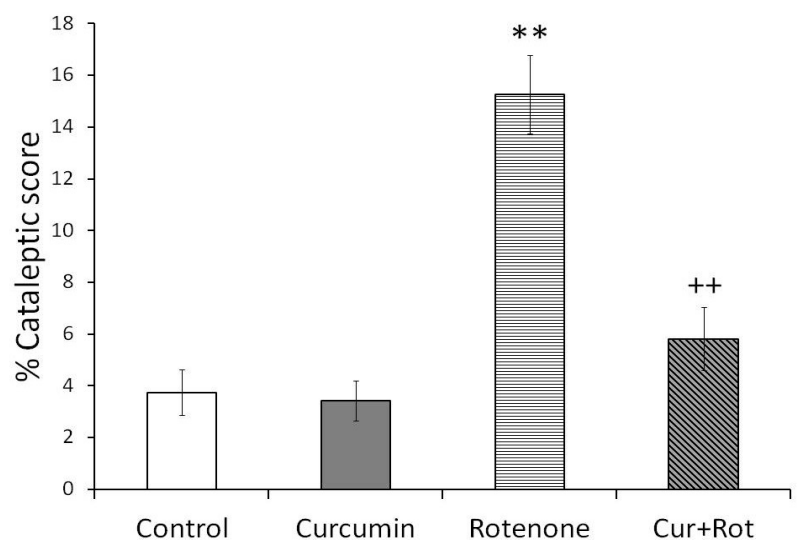

Fig. 5. Effect of prior administration of curcumin (100 $\mathrm{mg} / \mathrm{kg} /$ day) for 15 days on cataleptic behavior induced by rotenone observed in inclined plane apparatus. Values are mean + SD $(n=6)$. Data was analyzed by one-way ANOVA followed by Tukey's post-hoc test. Mean values were significantly different from controls $(* * p<0.01)$, and rotenone $(n=6)$ injected rats $(++p<0.01)$. The group that underwent successive rotenone administration for eight days exhibited a prominent cataleptic behavior as evident by significant increase in \% cataleptic score as compared to control rats. Pre-treatment with curcumin significantly normalized these observed parameters in Cur+Rot group. Cataleptic score in \% was calculated by (latency to move/ total time) x 100).

\section{Cataleptic and ambulatory behavior}

Figure 5 shows the effects of rotenone, curcumin and their co-administration on the induction of catalepsy. 
Statistical analysis by one-way ANOVA revealed significant effects of treatment on $\%$ cataleptic score $(F=143.66, p<0.01)$. Compared to control rats injected with rotenone exhibited a significant increase in cataleptic score $(p<0.01)$. Cur+Rot group showed significantly reduced cataleptic score $(p<0.01)$ compared to the group injected with rotenone alone. Rotenone also affected the ambulatory activity of rats as depicted in open field test (Fig. 6). One-way ANOVA for open field test showed significant effects of treatment on latency to move $(F=73.98, p<0.01)$ and number of square crossed $(F=7.89$, $p<0.01)$ in an open arena. Post-hoc analysis revealed that rotenone administration considerably increased latency to move $(p<0.01)$ and reduced the number of squares crossed
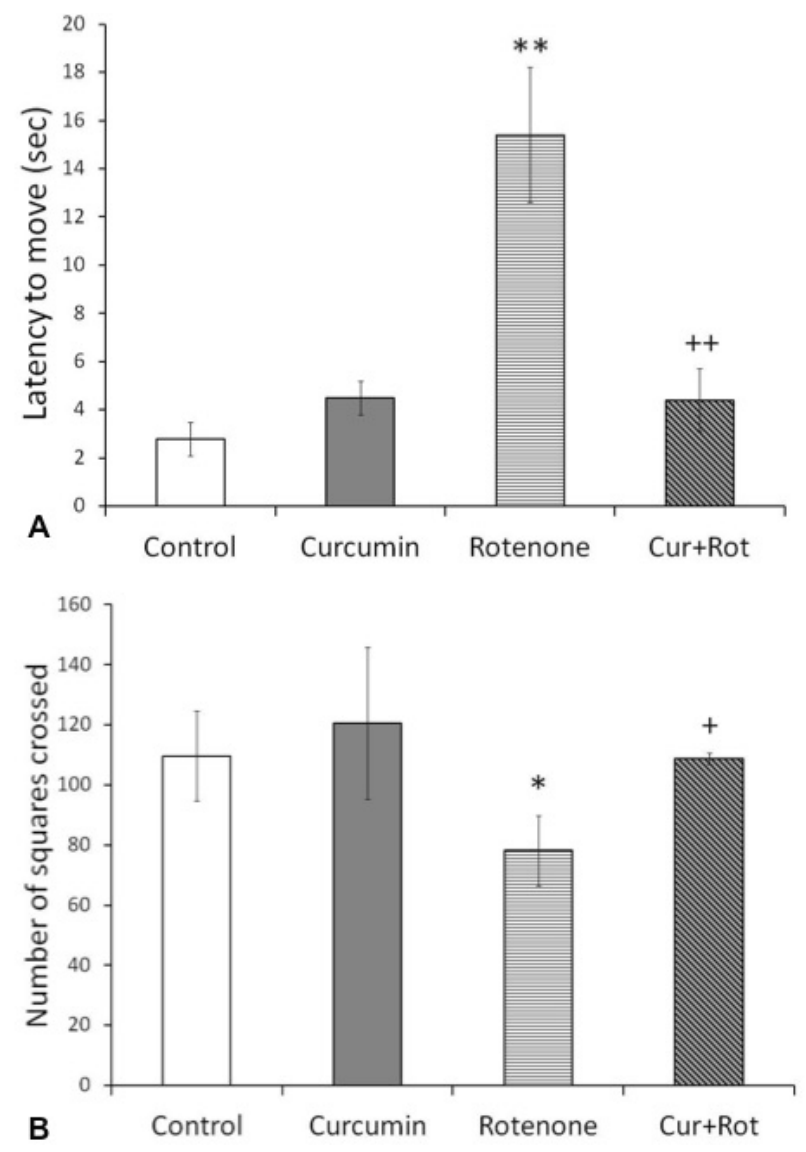

Fig. 6. Open field activity was performed to monitor the ambulatory activity in rats. Rotenone affected the motor skills of the rats and significantly decreased (A) latency to move, (B) number of squares crossed confirming the loss of motor activity. Values are represented as mean \pm SD $(n=6)$. Data was analyzed by Tukey's test following oneway ANOVA. Mean values were significantly different than that for controls $\left({ }^{* *} p<0.01,{ }^{*} p<0.05\right)$ and rotenone $(n=6)$ injected rats $(++p<0.01,+p<0.05)$. $(p<0.05)$ in open field as compared to that of control, Cur+Rot group exhibited significantly decreased latency to move $(p<0.01)$ and increased number of squares crossed $(p<0.05)$ as compared to rotenone group.

Rotenone administration exhibited a prominent loss of motor coordination in beam walking test (Fig. 7). In this test three beams of varying diameters $(3,2,1 \mathrm{~cm})$ were used and time to traverse the beam was monitored after the training session. One-way ANOVA revealed significant effects on the latency to cross the $3 \mathrm{~cm}$ beam $(F=20.17, p<0.01), 2 \mathrm{~cm}$ beam $(F=19.42, p<0.01)$ as well as $1 \mathrm{~cm}$ beam $(F=39.72, p<0.01)$. Rotenone administration significantly $(p<0.01)$ increased latency to cross the beam tested on all three sizes of beams showed by Post-hoc analysis. Whereas, latency to cross all the three sizes of beams was $(p<0.01)$ significantly reduced in Cur+Rot group as compared to that of rotenone injected group.

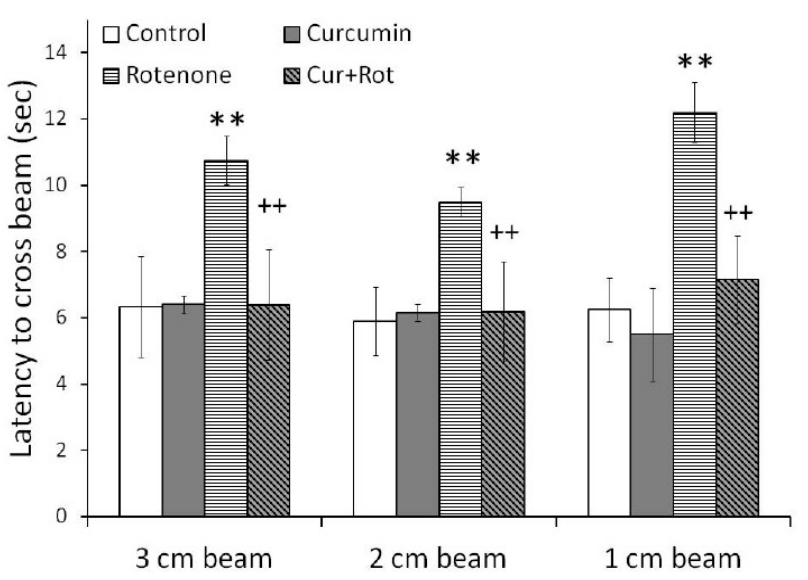

Fig. 7. Beam test was performed to assess the motor coordination. Subcutaneous injection of rotenone affected the motor coordination and significantly increased the time to cross the beam in all three beams of varying diameters as compared to control rats, confirming the loss of motor skills. Pre-treatment with curcumin significantly normalized the impaired motor activity in Cur+Rot group. Significant differences were obtained by one-way ANOVA followed by Tukey's post hoc test. $* * p<0.01$ as compared to controls and $++p<0.01$ when compared with rotenone injected group $(n=6)$. Values are mean $+\mathrm{SD}(\mathrm{n}=6)$.

\section{Gait behavior}

Walking pattern was also monitored by footprint test (Fig. 8A). There were significant effects of treatment on stride length of forelimb $(F=67.73, p<0.01)$ and hindlimb $(F=33.40, p<0.01)$, hind base width $(F=9.96$, $p<0.01)$ and paw overlapping $(F=27.30, p<0.01)$. In the present study administration of rotenone showed gait abnormalities as evident by significant shortened 

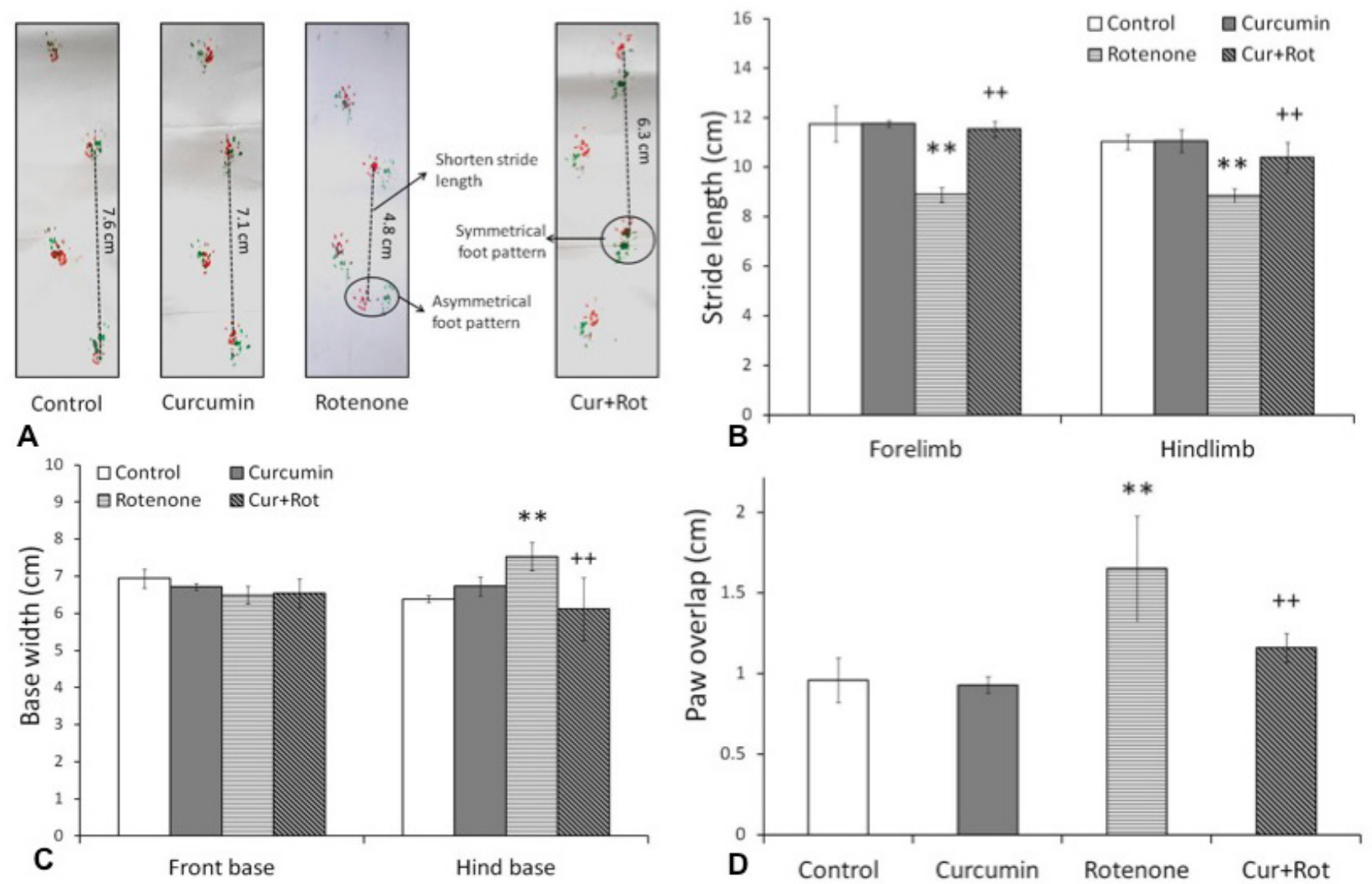

Fig. 8. Foot pattern of rats was attained by footprint test. (A) representation of footprint pattern analysis. Forepaws and hindpaws were coated with red and green ink, respectively. The dotted lines indicate stride length. The irregular distance between the foreand hindpaws in Rot administered rat is encircled whereas symmetrical foot pattern is encircled in Cur+Rot administered rat. The quantification of strides (B), base width (C) and paw overlap (D) in control $(n=6)$ and test $(n=6)$ groups. Values are mean \pm SD $(n=6)$. Data was analyzed by one-way ANOVA followed by Tukey's post-hoc test. Mean values were significantly different from controls $(* * p<0.01)$, and rotenone $(n=6)$ injected rats $(++p<0.01)$.

$(p<0.01)$ stride length for both forelimb and hindlimb as compared to that of controls (Fig. 8B). Hind base width (Fig. 8C) and paw overlapping (Fig. 8D) were also significantly increased $(p<0.01)$ in rotenone injected rats. Pre-administration of curcumin in rotenone injected rats showed normal walking pattern. In Cur+Rot group, forelimb stride and hindlimb stride were considerably increased $(p<0.01)$ than that of rotenone injected animals. Moreover, in Cur+Rot group hind base width and paw overlapping were significantly $(p<0.01)$ lower than rotenone group.

\section{Striatal monoamine levels}

Striatal DA and DOPAC levels were also determined in the present study. Data of standard and striatal brain samples were analyzed by the HPLC (Fig. 9A, B). DA $(F=68.56, p<0.01)$ and DOPAC $(F=23.30, p<0.01)$ levels were significantly affected by rotenone and curcumin treatment (Fig. 9C). Rotenone administration significantly reduced the DA $(p<0.01)$ and DOPAC $(p<0.01)$ levels while compared to control rats. Curcumin pre-treatment significantly attenuated the rotenone-induced decreased dopaminergic function in striatum as evident by a notably increase in DA $(p<0.01)$ and DOPAC $(p<0.01)$ levels as compared to the rats injected with rotenone alone.

\section{Brain cholinergic function}

In the current study striatal AChE activity and ACh levels were also estimated following the administration of rotenone and curcumin (Fig. 10). One-way ANOVA revealed significant effects of treatment on AChE activity $(F=33.41, p<0.01)$ and ACh levels $(F=33.41, p<0.01)$. Post-hoc analysis by Tukey's test showed that the rotenone administration significantly reduced $(p<0.01)$ AChE activity in brain. Whereas ACh content was significantly increased $(p<0.01)$ following the administration of rotenone. On the other hand, a significant increase in $\mathrm{AChE}$ 
activity $(p<0.01)$ and decrease in ACh levels $(p<0.01)$ was observed in Cur+Rot group as compared to that of rotenone injected rats.
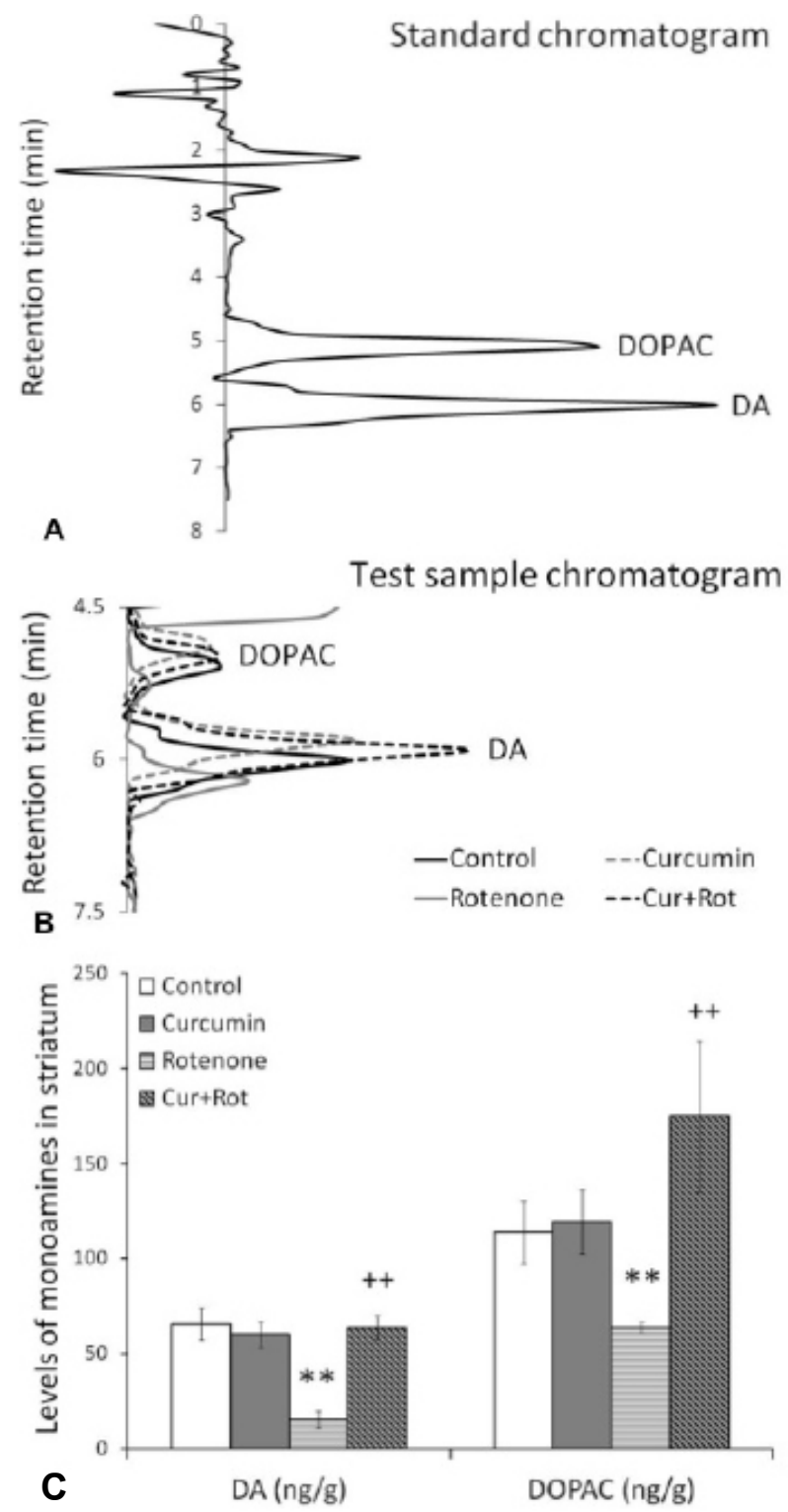

Fig. 9. The retention time of dopamine (DA) and dihydroxyphenyl acetic acid (DOPAC) in standard solution (A) and striatal brain region (B) of rats representing by HPLC chromatogram. Effect of prior administration of curcumin $(100 \mathrm{mg} / \mathrm{kg} / \mathrm{day})$ for 15 days on neurotransmitter levels (C). DA (ng/g of brain tissue) and DOPAC ( $\mathrm{ng} / \mathrm{g}$ of brain tissue) levels were analyzed at the end of present study. Significant differences were obtained by one-way ANOVA following Tukey's test. ${ }^{* *} p<0.01$ versus controls and $++p<0.01$ versus rotenone injected group $(n=6)$.

\section{Oxidative stress}

Another potential consequence of rotenone-induced toxicity is oxidative stress (Fig. 11). Statistical analysis showed significant effects of treatment on brain MDA $(F=29.05, p<0.01)$ and GSH $(F=57.12, p<0.01)$ levels (Fig. 11A). Rotenone injected rats exhibited a significant increase in MDA levels $(p<0.01)$ and significant decrease in GSH levels $(p<0.01)$ in brain as compared to that of controls. Whereas, pre-administration of curcumin significantly decreased MDA levels $(p<0.01)$ and increased GSH levels in Cur+Rot group when compared to that of rotenone group. To further test the relevance of rotenoneinduced oxidative damage, we investigated enzyme activity of GPx, SOD, and CAT (Fig. 11B). One-ANOVA revealed that there was a significant effect of treatment on GPx $(F=20.86, p<0.01)$, SOD $(F=15.61, p<0.01)$, and CAT $(F=54.73, p<0.01)$ activity in brain. GPx $(p<0.01)$ and CAT $(p<0.01)$ activity were significantly augmented whereas SOD activity was significantly reduced in rotenone injected rats compared to controls. Curcumin exhibits antioxidant property which was also revealed in the current study as evident by reduced MDA $(p<0.01)$ and increased GSH $(p<0.01)$ levels when compared with that of rotenone injected rats. Reduced GPx activity $(p<0.01)$, CAT activity $(p<0.01)$ and increased SOD activity $(p<0.01)$ were also observed in rotenone administered rats that were supplemented pre- with curcumin compared to rats treated with rotenone alone.

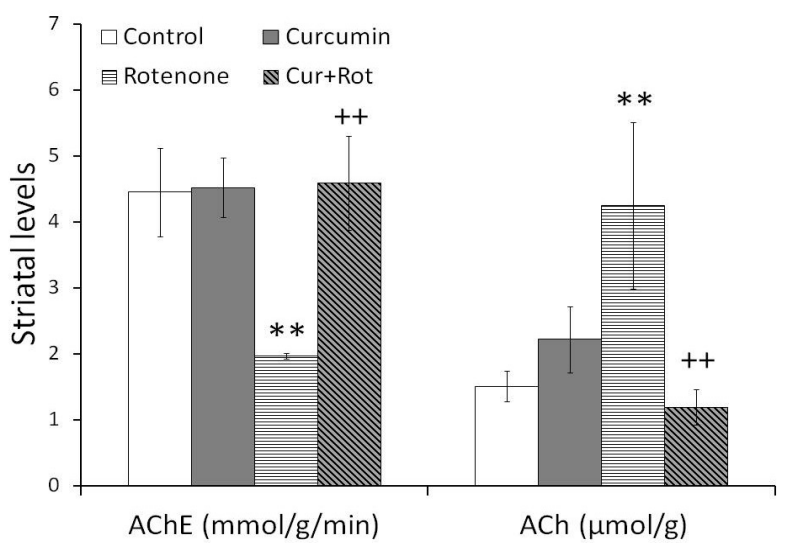

Fig. 10. Effect of rotenone and curcumin pre-treatment on striatal ACh $(\mu \mathrm{mol} / \mathrm{g})$ levels and $\mathrm{AChE}(\mathrm{mmol} / \mathrm{g} / \mathrm{min})$ activity. Significant differences were obtained by one-way ANOVA following Tukey's test. $* * p<0.01$ versus controls and $++p<0.01$ versus rotenone injected group $(n=6)$.

\section{DISCUSSION}

In the present study, behavioral results indicated that the administration of rotenone significantly decreased 
motor activity and increased rigidity in rats. The findings are consistent with the previous study which has demonstrated that motor impairments following rotenone administration (Alam and Schmidt, 2002; Khatri and Juvekar, 2016; Madiha et al., 2017).

In current study, curcumin treatment at the dose of $100 \mathrm{mg} / \mathrm{kg} /$ day in Cur+Rot treated rats produced significant augmentation in locomotor function which is in line with previous studies (Khatri and Juvekar, 2016; Mansouri et al., 2012). In Cur+Rot group, treatment with curcumin produced significant improvement in muscular function which is in accordance with previous study which reported that curcumin alleviated dystrophic muscle pathology and enhanced muscle strength (Pan et al., 2008).

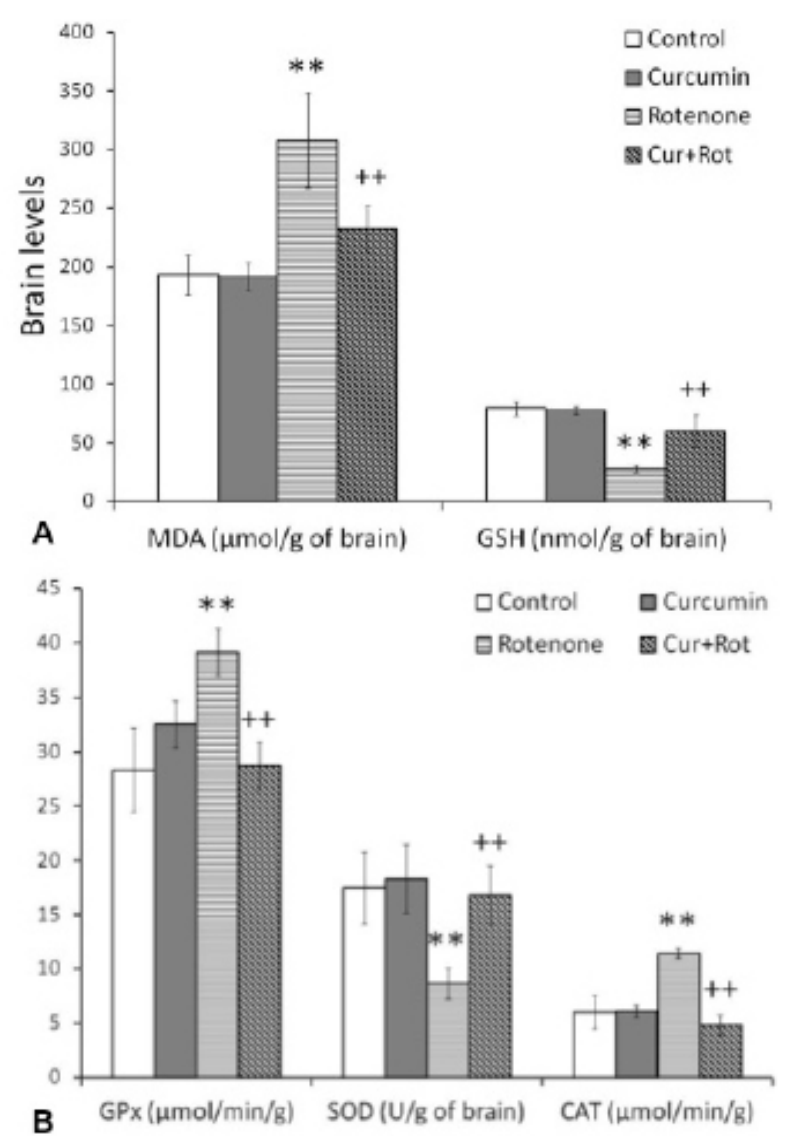

Fig. 11. Estimated MDA ( $\mu \mathrm{mol} / \mathrm{g}$ of brain tissue) (A) and $\mathrm{GSH}$ (nmol/g of brain tissue) levels (B) various enzyme activities followed by s.c. injection of rotenone. Enzymes activity such as SOD (U/g of brain), CAT ( $\mu \mathrm{mol} / \mathrm{min} / \mathrm{g}$ of brain tissue), and GPx ( $\mu \mathrm{mol} / \mathrm{min} / \mathrm{g}$ of brain tissue) were also estimated to determine the effects of 15 days curcumin pre-treatment on rotenone-induced oxidative stress. Significant differences were obtained by one-way ANOVA following Tukey's test. $* * p<0.01$ versus controls and $++p<0.01$ versus rotenone injected group $(n=6)$.
In our study footprint analysis showed that gait dynamics were significantly different in rotenone treated rats than curcumin treated rats. As stride length was significantly decreased in rotenone treated rats. On the other hand, rats in Cur+Rot group displayed a significant increase in stride length and motor symmetries showing restorative effects of curcumin on rotenone-induced gait disturbances and muscular response. The present findings demonstrated that rotenone-induced locomotor deficits were also reversed in Cur+Rot treated rats which further confirm the previous findings (Khatri and Juvekar, 2016). Neurochemical analysis was also done in this study which showed that administration of rotenone decreased DA levels (Madiha et al., 2017). This decrease in DA indicates the motor disorder associated with DA loss in striatum (Nehru et al., 2008). Therefore, all locomotor deficits observed in our study may be attributed to the decline in levels of DA in striatum. These alterations in DA and DOPAC in striatum were reversed by treatment with curcumin as observed in Cur+Rot group showed increased levels of DA and DO$\mathrm{PAC}$ in striatum. Our data is an agreement with the prior findings in which increase in DA and DOPAC levels were observed following curcumin administration (Rajeswari and Sabesan, 2008). Studies have been reported that curcumin improves DA levels (Zbarsky et al., 2005; Mythri et al., 2011; Yang et al., 2008).

In the present work, rotenone administration significantly decreased AChE activity which significantly increased ACh levels in these rats. This decrease in AChE activity following rotenone administration was attenuated by curcumin treatment which may be linked to restoration of cholinergic activity resulting in observed normalized ACh levels. ACh plays an important role in motor control in the central nervous systems (Abdel-Salam et al., 2014). The transmitted signal is terminated by AChE in cholinergic neurotransmission. Study showed that pesticides including rotenone act as an $\mathrm{AChE}$ inhibitor and reduced the level of AChE enzyme which is in agreement with our study (Abdel-Salam et al., 2014; Madiha and Haider, 2017). In movement control DA acts as inhibitory neurotransmitter in basal ganglia and ACh acts as excitatory neurotransmitter. Due to decreased DA levels inhibitory influences are lost and excitatory mechanisms are overactivated. Increase in ACh levels due to lack of DA level in our study may be the cause of observed motor deficits in rotenone treated rats (Obeso et al., 2008). It may be suggested that in the present study treatment with curcumin activated the inhibitory mechanism and lowered the excitatory mechanisms as a result of which there was increase in DA levels which concomitantly normalized the levels of ACh and AChE activity. Therefore administration of curcumin in present study restores the cholinergic 
functions resulting in attenuation of typical behavior such as catalepsy and tremors which strengthens the protective role of curcumin against cholinergic and dopaminergic imbalance which has not been studied earlier.

Oxidative stress produced as a consequence of mitochondrial dysfunction and oxidative metabolism of DA (Schapira, 2005). The production of reactive oxygen species (ROS) and nitrogen species is remunerated by antioxidant mechanisms (e.g., CAT, GPx, SOD and GSH) (Halliwell, 2001). When these antioxidant mechanisms are overwhelmed by free radical this produces oxidative stress which might result in cellular and tissue damage (Halliwell, 2001). In the present study rotenone administration might produce oxidative stress via excess generation of free radicals (Yanpallewar et al., 2004). Different studies have pointed out that rotenone exposure reduces complex I activity and produces oxidative stress (Votyakova and Reynolds, 2001; Moon et al., 2005). The present results are consistent with the previous reports showing decline in GSH level, SOD activity and increased LPO level, and CAT, GPx activities following rotenone exposure (Yanpallewar et al., 2004; Sharma and Nehru, 2013). It is well reported that increase in MDA levels is taken as biomarker of oxidative stress (Pearce et al., 1997). MDA is the most cytotoxic aldehyde formed in the process of LPO. Our results showed that rotenone increased LPO (Hensley et al., 1998; Mishra et al., 2004). It is well established that SOD firstly can work against oxidative stress and it's only possible when it is pursued by activities of GPx and CAT (Bhattacharya et al., 2001). It is well documented that decrease in GSH and increase in GPx activity would decrease the capacity of neuron to detoxify $\mathrm{H}_{2} \mathrm{O}_{2}$ and increase the free radical formation which ultimately leads LPO as indicated by high MDA levels. Curcumin crosses the blood brain barrier, and detoxifies the reactive species and thus provides protection against LPO (Iwunze and McEwan, 2004). In present study curcumin mediated antioxidant mechanism provides protection from rotenone toxicity by lesser utilization of GSH, and SOD which act as first line of defence and result in decreased level of LPO (Raza et al., 2008). It is well established that oxidative stress can vary the neurotransmission by damaging the membrane integrity (Bouayed et al., 2009). Curcumin being a free radical scavenger (Iwunze and McEwan, 2004), prevents the cell damage by binding to the free radicals and neutralizing its unpaired electron is able to prevent the damage to dopaminergic neurons caused by rotenone. It has been shown that pre-treatment with curcumin prior to the administration of toxin might increase the density of dopaminergic neurons (Zbarsky et al., 2005; Vajragupta et al., 2003). In the present study curcumin appear to ameliorate the rotenone-induced oxidative stress by restoring the SOD activity and normalizing the CAT and GPx activity which eventually restore the GSH levels. All these aforesaid effects would increase the capacity of neuron to detoxify free radicals which may be attributed to decreased LPO and hence reversal of PD-like symptoms.

\section{CONCLUSION}

In conclusion, the findings of the present study provide evidence that daily administration of rotenone for eight days considerably induced toxicity as manifested by behavioral deficits, biochemical, and neurochemical changes in rats. Pre-treatment with curcumin reversed the locomotor deficits. The biochemical and neurochemical alterations produced by rotenone administration were also restored. Curcumin pre-treatment may have altered the bioavailability of rotenone and its efficiency to inhibit mitochondrial complex I. The present study suggests that curcumin can show beneficial effects in relieving the symptoms of Parkinson's disease. These protective effects may be attributed to the antioxidant effects of curcumin. Our study is the first comprehensive study highlighting towards a deeper understanding of protective effects of curcumin on rotenone-induced behavioral deficits, dopaminergic and cholinergic imbalance and antioxidant enzyme activity alterations. This study might draw more attention towards cholinergic and dopaminergic neurotransmission and protective role of curcumin in restoring cholinergic and dopaminergic functions. However, more work is required to give ultimate evidence of disease-modifying capabilities of curcumin. Histopathological examination is needed to further validate beneficial effect of curcumin on rotenone-induced toxicity in rats.

\section{ACKNOWLEDGEMENT}

University of Karachi, Karachi, Pakistan funded this project.

\section{Statement of conflict of interest}

Nothing to declare.

\section{REFERENCES}

Abdel-Salam, O.M.E., Omara, E.A., Youness, E.R., Khadrawy, Y.A., Mohammed, N.A. and Sleem, A.A., 2014. Rotenone-induced nigrostriatal toxicity is reduced by methylene blue. J. Neurorestoratol., 2: 65-80. https://doi.org/10.2147/JN.S49207

Alam, M. and Schmidt, W.J., 2002. Rotenone destroys dopaminergic neurons and induces parkinsonian symptoms in rats. Behav. Brain Res., 136: 317-324. 
https://doi.org/10.1016/S0166-4328(02)00180-8

Batool, F., Haleem, M.A. and Haleem, D.J., 2010. Acute administration of clozapine and risperidone altered dopamine metabolism more in rat caudate than in nucleus accumbens a dose-response relationship. Sci. Pharm., 78: 259-274. https://doi.org/10.3797/ scipharm.0907-20

Batool, Z., Sadir, S., Liaquat, L., Tabassum, S., Madiha, S., Rafiq, S., Tariq, S., Saleem, S., Naqvi, F., Perveen, T. and Haider, S., 2016. Repeated administration of almonds increases brain acetylcholine levels and enhances memory function in healthy rats while attenuates memory deficits in animal model of amnesia. Brain Res. Bull., 120: 63-74. https://doi. org/10.1016/j.brainresbull.2015.11.001

Betarbet, R., Sherer, T.B., MacKenzie, G., GarciaOsuna, M., Panov, A.V. and Greenamyre, J.T., 2000. Chronic systemic pesticide exposure reproduces features of Parkinson's disease. Nature Neurosci., 3: 1301-1306. https://doi.org/10.1038/81834

Bhattacharya, A., Ghosal, S. and Bhattacharya, S.K., 2001. Anti-oxidant effect of Withania somnifera glycowithanolides in chronic footshock stressinduced perturbations of oxidative free radical scavenging enzymes and lipid peroxidation in rat frontal cortex and striatum. J. Ethnopharmacol., 74: 1-6. https://doi.org/10.1016/S03788741(00)00309-3

Bocarsly, M.E., Barson, J.R., Hauca, J.M., Hoebel, B.G., Leibowitz, S.F. and Avena, N.M., 2012. Effects of perinatal exposure to palatable diets on body weight and sensitivity to drugs of abuse in rats. Physiol. Behav., 107: 568-575. https://doi. org/10.1016/j.physbeh.2012.04.024

Bouayed, J., Rammal, H. and Soulimani, R., 2009. Oxidative stress and anxiety: Relationship and cellular pathways. Oxid. Med. Cell Longev., 2: 6367. https://doi.org/10.4161/oxim.2.2.7944

Costall, B. and Naylor, R.J., 1974. On catalepsy and catatonia and the predictability of the catalepsy test for neuroleptic activity. Psychopharmacologia, 34: 233-241.

Freire, C. and Koifman, S., 2012. Pesticide exposure and Parkinson's disease: Epidemiological evidence of association. Neurotoxicology, 33: 947-971. https://doi.org/10.1016/j.neuro.2012.05.011

$\mathrm{Fu}$, S. and Kurzrock, R., 2010. Development of Curcumin as an epigenetic agent. Cancer, 116: 4670-4676. https://doi.org/10.1002/cncr.25414

Gatto, N.M., Cockburn, M., Bronstein, J., Manthripragada, A.D. and Ritz, B.B., 2009. Wellwater consumption and Parkinson's disease in rural
California. Environ. Hlth. Perspect., 117: 19121918. https://doi.org/10.1289/ehp.0900852

Greenamyre, J.T., Cannon, J.R., Drolet, R. and Mastroberardino, P.G., 2011. Lessons from the rotenone model of Parkinson's disease. Trends Pharmacol. Sci., 31: 141-143. https://doi. org/10.1016/j.tips.2009.12.006

Haider, S., Naqvi, F., Batool, Z., Tabassum, S., Sadir, S., Liaquat, L., Naqvi, F., Zuberi, N.A., Shakeel, H. and Perveen, T., 2015. Pretreatment with curcumin attenuates anxiety while strengthens memory performance after one short stress experience in male rats. Brain Res. Bull., 115: 1-8. https://doi. org/10.1016/j.brainresbull.2015.04.001

Haider, S., Saleem, S., Perveen, T., Tabassum, S., Batool, Z., Sadir, S., Liaquat, L. and Madiha, S., 2014. Age-related learning and memory deficits in rats: Role of altered brain neurotransmitters, acetylcholinesterase activity and changes in antioxidant defense system. Age (Dordr.), 36: 12911302. https://doi.org/10.1007/s11357-014-9653-0

Haleem, D.J., 1990. Injected TRP increases brain but not plasma tryptophan levels more in ethanol treated rats. Life Sci. (USA), 47: 971-979. https:// doi.org/10.1016/0024-3205(90)90544-2

Haleem, D.J. and Perveen, T., 1994. Brain regional serotonin synthesis following adaptation to repeated restraint. Neuro Rep., 5: 1785-1788. https://doi. org/10.1097/00001756-199409080-00025

Haleem, D.J., Samad, N. and Haleem, M.A., 2007. Reversal of haloperidol-induced VCMs and supersensitive somatodendritic serotonergic response by buspirone in rats. Pharmacol. Biochem. Behav., 87: 115-121. https://doi.org/10.1016/j. pbb.2007.04.007

Haider, S., Tabassum, S. and Perveen, T., 2016. Scopolamine induced greater alterations in neurochemical profile and increased oxidative stress demonstrated a better model of dementia: A comparative study. Brain Res. Bull., 127: 234-247.

Halliwell, B., 2001. Role of free radicals in the neurodegenerative diseases: therapeutic implications for antioxidant treatment. Drugs Aging, 18: 685-716. https://doi.org/10.2165/00002512200118090-00004

Hatcher, H., Planalp, R., Cho, J., Torti, F.M. and Torti, S.V., 2008. Curcumin: from ancient medicine to current clinical trials. Cell Mol. Life Sci., 65: 16311652. https://doi.org/10.1007/s00018-008-7452-4

Hatefi, Y., 1985. The mitochondrial electron transport and oxidative phosphorylation system. Annu. Rev. Biochem., 54: 1015-1069. https://doi.org/10.1146/ 
annurev.bi.54.070185.005055

Hensley, K., Pye, Q.N., Maidt, M.L., Stewart, C.A., Robinson, K.A., Jaffrey, F. and Floyd, R.A., 1998. Interaction of alpha-phenyl-N-tert-butyl nitrone and alternative electron acceptors with complex I indicates a substrate reduction site upstream from the rotenone binding site. $J$ Neurochem., 71: 2549-2557. https://doi.org/10.1046/j.14714159.1998.71062549.x

Iwunze, M.O. and McEwan, D., 2004. Peroxynitrite interaction with curcumin solubilized in ethanolic solution. Cell Mol. Biol. (Noisy-le-grand), 50: 749752.

Jover, R., Rodrigo, R., Felipo, V., Insausti, R., SáezValero, J., García-Ayllón, M.S., Suárez, I., Candela, A., Compañ, A., Esteban, A., Cauli, O., Ausó, E., Rodríguez, E., Gutiérrez, A., Girona, E., Erceg, S., Berbel, P. and Pérez-Mateo, M., 2006. Brain edema and inflammatory activation in bile duct ligated rats and diet-induced hyperammonemia: A model of Hepatic encephalopathy in cirrhosis. Hepatology, 43: 1257-1266. https://doi.org/10.1002/hep. 21180

Khatri, D.K. and Juvekar, A.R., 2016. Neuroprotective effect of curcumin as evinced by abrogation of rotenone-induced motor deficits, oxidative and mitochondrial dysfunctions in mouse model of Parkinson's disease. Pharmacol. Biochem. Behav., 150-151: 39-47. https://doi.org/10.1016/j. pbb.2016.09.002

Kondziela, W., 1964. Anew method for the measurement of muscle relaxation in white mice. Arch. Int. Pharmacodyn. Ther., 152: 277-284.

Madiha, S. and Haider, S., 2017. Imbalance between dopaminergic and cholinergic neurotransmission following rotenone administration suggestive of Parkinson's-like symptoms in male rats [abstract]. Mov Disord., 32(Suppl 2): S278-S280.

Madiha, S., Tabassum, S., Batool, Z., Liaquat, L., Sadir, S., Shahzad, S., Perveen, T. and Haider, S., 2017. Assessment of gait dynamics in rotenone-induced rat model of Parkinson's disease by footprint method. Pak. J. pharm. Sci., 30: 943-948.

Mansouri, Z., Sabetkasaei, M., Moradi, F., Masoudnia, F. and Ataie, A., 2012. Curcumin has neuroprotection effect on homocysteine rat model of Parkinson. J. Mol. Neurosci., 47: 234-242. https://doi. org/10.1007/s12031-012-9727-3

Mishra, B., Priyadarsini, K.I., Bhide, M.K., Kadam, R.M. and Mohan, H., 2004. Reactions of superoxide radicals with curcumin: probable mechanisms by optical spectroscopy and EPR. Free Radic. Res., 38: 355-362. https://doi.org/10.1080/1071576031
0001660259

Moon, Y., Lee, K.H., Park, J.H., Geum, D. and Kim, K., 2005. Mitochondrial membrane depolarization and the selective death of dopaminergic neurons by rotenone: Protective effect of coenzyme Q10. J. Neurochem., 93: 1199-1208. https://doi. org/10.1111/j.1471-4159.2005.03112.x

Mythri, R.B., Harish, G., Dubey, S.K., Misra, K. and Bharath, M.M., 2011. Glutamoyl diester of the dietary polyphenol curcumin offers improved protection against peroxynitrite-mediated nitrosative stress and damage of brain mitochondria in vitro: Implications for Parkinson's disease. Mol. Cell. Biochem., 347: 135-143. https://doi. org/10.1007/s11010-010-0621-4

Nascimento-Ferreira, I., Nóbrega, C., VasconcelosFerreira, A., Onofre, I., Albuquerque, D., Aveleira, C., Hirai, H., Déglon, N. and Pereira de Almeida, L., 2013. Beclin 1 mitigates motor and neuropathological deficits in genetic mouse models of Machado-Joseph disease. Brain, 136: 21732188. https://doi.org/10.1093/brain/awt144

Nehru, B., Verma, R., Khanna, P. and Sharma, S.K., 2008. Behavioral alterations in rotenone model of Parkinson's disease: attenuation by co-treatment of centrophenoxine. Brain Res., 1201: 122-127. https://doi.org/10.1016/j.brainres.2008.01.074

Obeso, J.A., Rodríguez-Oroz, M.C., Benitez-Temino, B., Blesa, F.J., Guridi, J., Marin, C. and Rodriguez, M., 2008. Functional organization of the basal ganglia: Therapeutic implications for Parkinson's disease. Mov. Disord., 23: S548-S559. https://doi. org/10.1002/mds.22062

Pan, Y., Chen, C., Shen, Y., Zhu, C.H., Wang, G., Wang, X.C., Chen, H.Q. and Zhu, M.S., 2008. Curcumin alleviates dystrophic muscle pathology in $\mathrm{mdx}$ mice. Mol. Cells, 25: 531-537.

Pearce, R.K., Owen, A., Daniel, S., Jenner, P. and Marsden, C.D., 1997. Alterations in the distribution of glutathione in the substantia nigra in Parkinson's disease. J. Neural Transm., 104: 661-677. https:// doi.org/10.1007/BF01291884

Rajeswari, A., 2006. Curcumin protects mouse brain from oxidative stress caused by 1-methyl-4phenyl-1,2,3,6-tetrahydropyridine. Eur. Rev. Med. Pharmacol. Sci., 10: 157-161.

Rajeswari, A. and Sabesan, M., 2008. Inhibition of monoamine oxidase-B by the polyphenolic compound, curcumin and its metabolite tetrahydrocurcumin, in a model of Parkinson's disease induced by MPTP neurodegeneration in mice. Inflammopharmacology, 16: 96-99. https:// 
doi.org/10.1007/s10787-007-1614-0

Raza, H., John, A., Brown, E.M., Benedict, S. and Kambal, A., 2008. Alterations in mitochondrial respiratory functions, redox metabolism and apoptosis by oxidant 4-hydroxynonenal and antioxidants curcumin and melatonin in PC12 cells. Toxicol. appl. Pharmacol., 226: 161-168. https:// doi.org/10.1016/j.taap.2007.09.002

Schapira, A.H.V., 2005. Present and future drug treatment for Parkinson's disease. J. Neurol. Neurosurg. Psychiat., 76: 1472-1478. https://doi. org/10.1136/jnnp.2004.035980

Sharma, N. and Nehru, B., 2013. Beneficial effect of vitamin $\mathrm{E}$ in rotenone induced model of PD: Behavioural, neurochemical and biochemical study. Exp. Neurobiol., 22: 214-223. https://doi. org/10.5607/en.2013.22.3.214

Vajragupta, O., Boonchoong, P., Watanabe, H., Tohda, M., Kummasud, N. and Sumanont, Y., 2003. Manganese complexes of curcumin and its derivatives: evaluation for the radical scavenging ability and neuroprotective activity. Free Radic. Biol. Med., 35: 1632-1644. https://doi. org/10.1016/j.freeradbiomed.2003.09.011

Votyakova, T.V. and Reynolds, I.J., 2001. DeltaPsi (m) Dependent and -independent production of reactive oxygen species by rat brain mitochondria. $J$. Neurochem., 79: 266-277. https://doi.org/10.1046/ j.1471-4159.2001.00548.x

Yang, S., Zhang, D., Yang, Z., Hu, X., Qian, S., Liu, J., Wilson, B., Block, M. and Hong, J.S., 2008. Curcumin perotects dopaminergic neuron against LPS induced neurotoxicity in primary rat neuron/ glia culture. Neurochem. Res., 33: 2044-2053. https://doi.org/10.1007/s11064-008-9675-z

Yanpallewar, S.U., Rai, S., Kumar, M. and Acharya, S.B., 2004. Evaluation of antioxidant and neuroprotective effect of Ocimum sanctum on transient cerebral ischemia and long-term cerebral hypoperfusion. Pharmacol. Biochem. Behav., 79: 155-164. https:// doi.org/10.1016/j.pbb.2004.07.008

Zbarsky, V., Datla, K.P., Parkar, S., Rai, D.K., Aruoma, O.I. and Dexter, D.T., 2005. Neuroprotective properties of the natural phenolic antioxidants curcumin and naringenin but not quercetin and fisetinin a 6-OHDA model of Parkinson's disease. Free Radic. Res., 39: 1119-1125. https://doi. org/10.1080/10715760500233113

Zeb, Q., Naeem, M., Khan, S.A. and Ahmad, S., 2016. Effect of insecticides on the population of aphids, natural enemies and yield components of wheat. Pakistan J. Zool., 48: 1839-1848. 